\title{
Circuit
}

Musiques contemporaines

\section{Anamorphoses (essai sonore)}

\section{Mario Gauthier}

Volume 26, numéro 3, 2016

URI : https://id.erudit.org/iderudit/1038519ar

DOI : https://doi.org/10.7202/1038519ar

Aller au sommaire du numéro

Éditeur(s)

Les Presses de l’Université de Montréal

ISSN

1183-1693 (imprimé)

1488-9692 (numérique)

Découvrir la revue

Citer ce document

Gauthier, M. (2016). Anamorphoses (essai sonore). Circuit, 26(3), 83-84. https://doi.org/10.7202/1038519ar

Ce document est protégé par la loi sur le droit d'auteur. L'utilisation des services d'Érudit (y compris la reproduction) est assujettie à sa politique d'utilisation que vous pouvez consulter en ligne.

https://apropos.erudit.org/fr/usagers/politique-dutilisation/
Cet article est diffusé et préservé par Érudit.

Érudit est un consortium interuniversitaire sans but lucratif composé de l'Université de Montréal, l'Université Laval et l'Université du Québec à Montréal. Il a pour mission la promotion et la valorisation de la recherche. https://www.erudit.org/fr/ 


\title{
Anamorphoses (essai sonore)
}

\author{
Mario Gauthier
}

Document sonore disponible en ligne sur le site web de Circuit:

www.revuecircuit.ca

J'ai tenté, dans ce podcast, de rendre compte d'une idée de Pierre Schaeffer selon laquelle

[le] cinéma et la radio n'ont pas seulement pour rôle de transmettre les images et les sons, mais de leur faire dire quelque chose. L'image d'un objet, la modulation d'un bruit, ne nous parviennent plus en tant que telles, non seulement à cause de la signification qui leur est attachée, mais à cause de la suggestion dont elles sont porteuses ${ }^{1}$.

Cette idée de «dire sans mots», de «suggérer sans montrer », c'est-à-dire de considérer que le son possède un aspect narratif et imagier inhérent, m’a toujours interpelé malgré la difficulté certaine qui se pose dès qu'il s'agit de « rendre compte» de cet effet.

Je suis conscient ici que «quelque chose dans l'esprit résiste à la phrase ${ }^{2}$ ", en ceci que cette proposition suggère qu'il existerait un vacillement préalable et continu entre l'entendu, le vu, le visible, l'imagier et l'imaginaire, et dont l'auditeur tiendrait compte de manière volontaire et inconsciente dans sa compréhension de ce «dire» qui leur est sous-jacent.

Afin de mettre ce phénomène en relief, j’ai conçu une série de jeux sonores d'allers-retours entre ces deux états, espérant ainsi rendre potentiellement audible comment agit ce processus «à double détente ${ }^{3} »$. Car si le son engendre intrinsèquement de l'imagier, on peut s'interroger quant aux formes que cela peut prendre. Quelle est la part de l'imaginaire du spectateur dans ce transfert de réalisme et d'affects? Opère-t-il à partir de résidus de conscience qui proposeraient la "possibilité de réaliser [un] savoir dans la matière intuitive qui [lui] est fournie ${ }^{4} »$ ? Que devient alors la causalité première? Etc.
1. Pierre Schaeffer (2010), Essai sur la radio et le cinéma : esthétique et technique des arts-relais, 1941-1942, Paris, Éditions Allia, p. 47.

2. Daniel Oster (2001), Rangements, Paris, P.O.L, p. 213.

3. C'est-à-dire "qui fait son effet en deux temps". On utilise ce terme en armurerie.

4. Jean-Paul Sartre (1948), L'imaginaire, psychologie phénoménologique de l'imagination, Paris, Gallimard, p. 42. 
5. Michel Chion (1983), Guide des objets sonores, Paris, INA/Buchet/ Chastel, p. 24.

6. Antonin Artaud en 1947. La phrase exacte est: "Et cet envers sera son véritable endroit." Finale de Pour en finir avec le jugement de dieu ", création radiophonique diffusée à l'ORTF. Disponible en ligne: <www.youtube. com/watch?v=TubmxQ7lkk8> (consulté le 14 octobre 2016).
Comme tout cela se passe simultanément dans un temps réel et un temps psychologique, la notion d'anamorphose, telle que Pierre Schaeffer la décrit en disant qu'elle est un « cas particulier de corrélation entre signal physique et objet sonore [...] faisant penser à une déformation psychologique de la réalité physique, et qui [traduit] l'irréductibilité de la perception à la mesure physique ${ }^{5}$, m’a semblé être adéquate pour rendre compte de ces phénomènes perceptifs liés à la fois au souvenir et à la perception subjective de ce temps aux rivages incertains.

Comment se construit notre perception? Comment - telles les anamorphoses justement - le son acquiert-il « un sens », par associations référentielles, prises en soi ou non? Comment se déploie-t-il en générant des jeux continus de sens dans un espace-temps donné, fictif ou réel? Ces quelques questions résument ce que j’ai cherché à rendre compte dans ce podcast dans lequel collages, créations et citations sont agglomérés en des formes parfois improbables, mais qui toutes, tentent de témoigner d'une sorte «d'envers de l'endroit ${ }^{6}$ ", celui «que disent» les choses, par-devers et en nous, en quelque sorte.

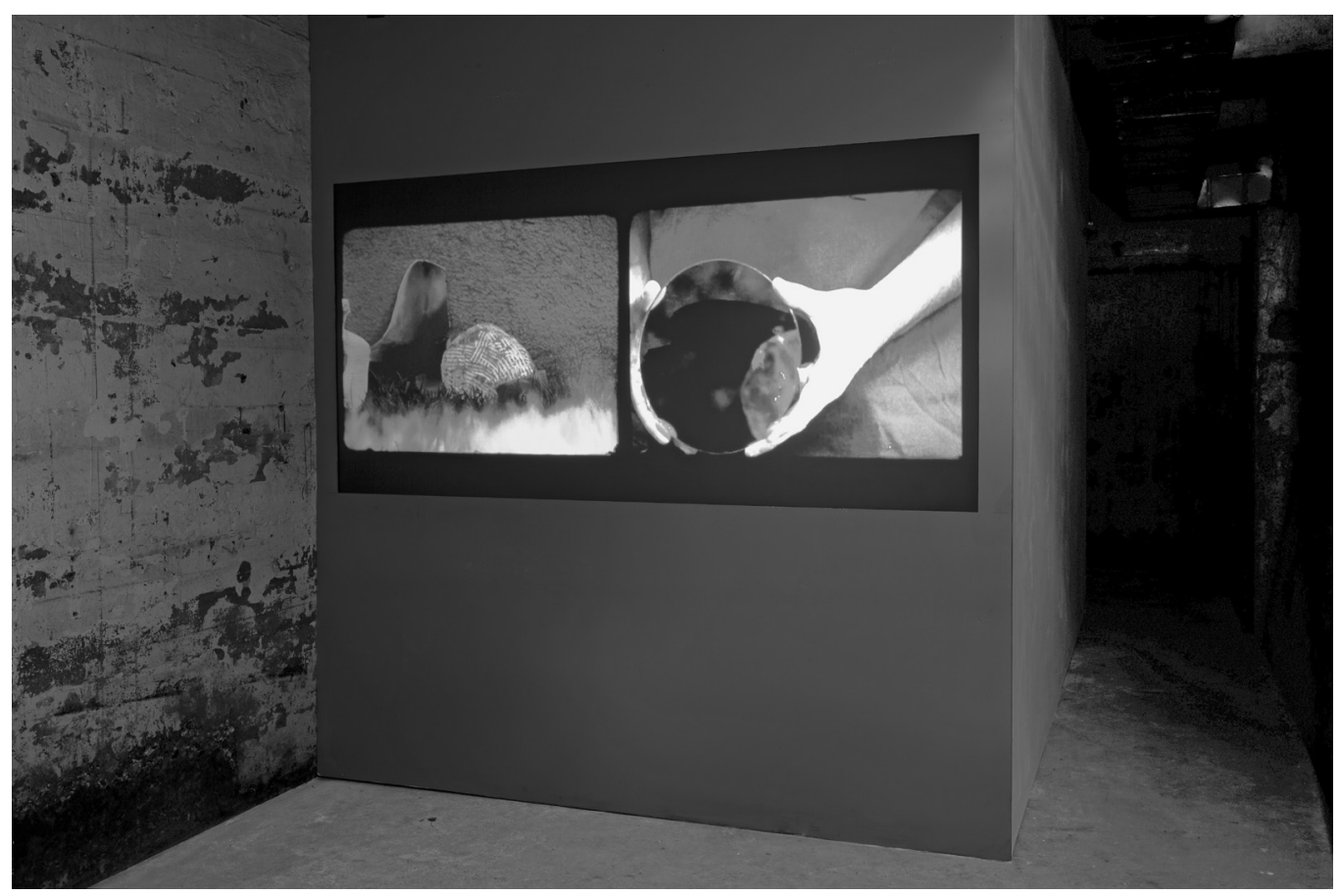

Steve Roden, Striations (Stones and Clouds), 2011. Encre et films 16 mm transférés sur vidéo, couleur, muet, 6 min. Image ca2011, SculptureCenter and the artists. Photo : Jason Mandella. 